\section{Essential function of LIF receptor in motor neurons}

\author{
Meng Li*, Michael Sendtner $\dagger$ \& Austin Smith*‡ \\ * Centre for Genome Research, University of Edinburgh, \\ King's Buildings, West Mains Road, Edinburgh EH9 3JQ, UK \\ † Clinical Research Unit for Neuroregeneration, Department of \\ Neurology, University of Würzburg, Josef-Schneider-Strasse 11, \\ 97080 Würzburg, Germany
}

DEVELOPMENT and maintenance of the mammalian nervous system is dependent upon neurotrophic cytokines. One class of neurotrophic factor acts through receptor complexes involving the lowaffinity leukaemia inhibitory factor receptor subunit (LIF-R) ${ }^{1-3}$. Members of this family of cytokines, such as ciliary neurotrophic factor (CNTF) and leukaemia inhibitory factor (LIF), have profound effects on the survival and maintenance of motor neurons ${ }^{4-10}$. Recently it was reported that mice lacking LIF-R die shortly after birth $^{11}$ unlike mice lacking CNTF or LIF which are viable. Here we describe histopathological analyses of lifr mutants that reveal a loss $>35 \%$ of facial motor neurons, $40 \%$ of spinal motor neurons and $50 \%$ of neurons in the nucleus ambiguus. These findings point to the existence of a ligand for LIF-R that is required for the normal development of motor neurons in both brainstem nuclei and spinal cord.

A deletion of $\sim 20 \mathrm{~kb}$ of the lifr gene was created by homologous recombination in embryonic stem (ES) cells (Fig. 1). The deleted sequence contains seven exons that encode most of the presumptive ligand-binding region. This was replaced by an internal ribosome entry site-lac $Z /$ neo cassette (IRES $\beta$ geopA $)^{12,13}$, thereby introducing a histochemical reporter into the locus. The mutant allele was established on hybrid 129/CBA and 129/MF1 backgrounds by appropriate matings of transmitting chimaeras.

Breeding of the mutated allele to homozygosity resulted in the production of liveborn homozygous pups on both genetic backgrounds. The mutant animals were smaller and had a $20 \%$ reduction in body weight $(1.33 \pm 0.22 \mathrm{~g}, n=7)$ compared with heterozygous $(1.67 \pm 0.19 \mathrm{~g}, n=18)$ and wild-type $(1.67 \pm 0.22 \mathrm{~g}$, $n=8$ ) littermates at birth. They lacked vigour, did not feed and rapidly became moribund. Although they exhibited limb movements, they were incapable of righting. In all cases so far, homozygotes have died within $24 \mathrm{~h}$ of birth and usually within the first $12 \mathrm{~h}$. Similar findings were recently described by Ware et al. ${ }^{\prime \prime}$.

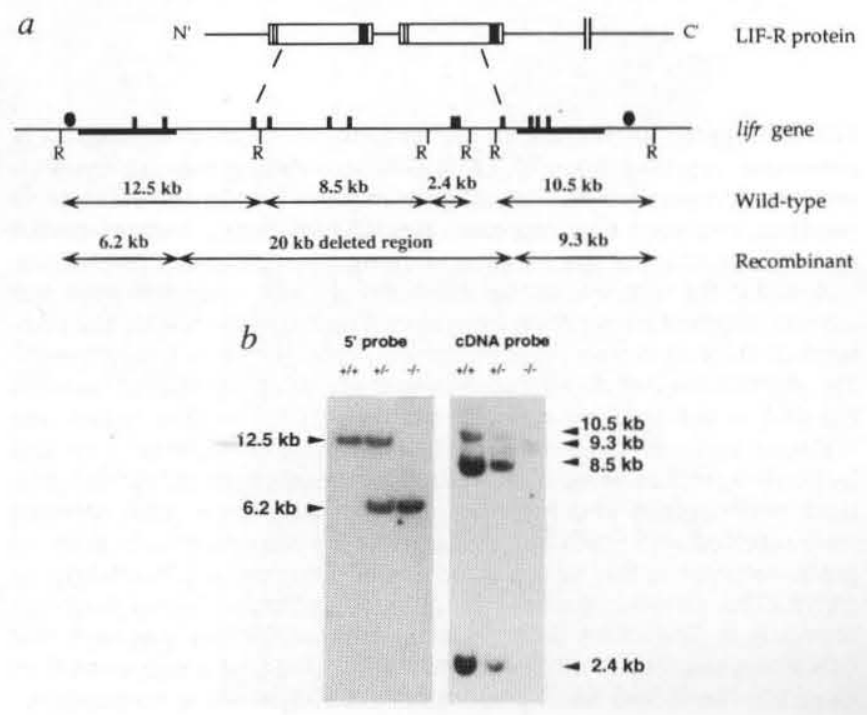



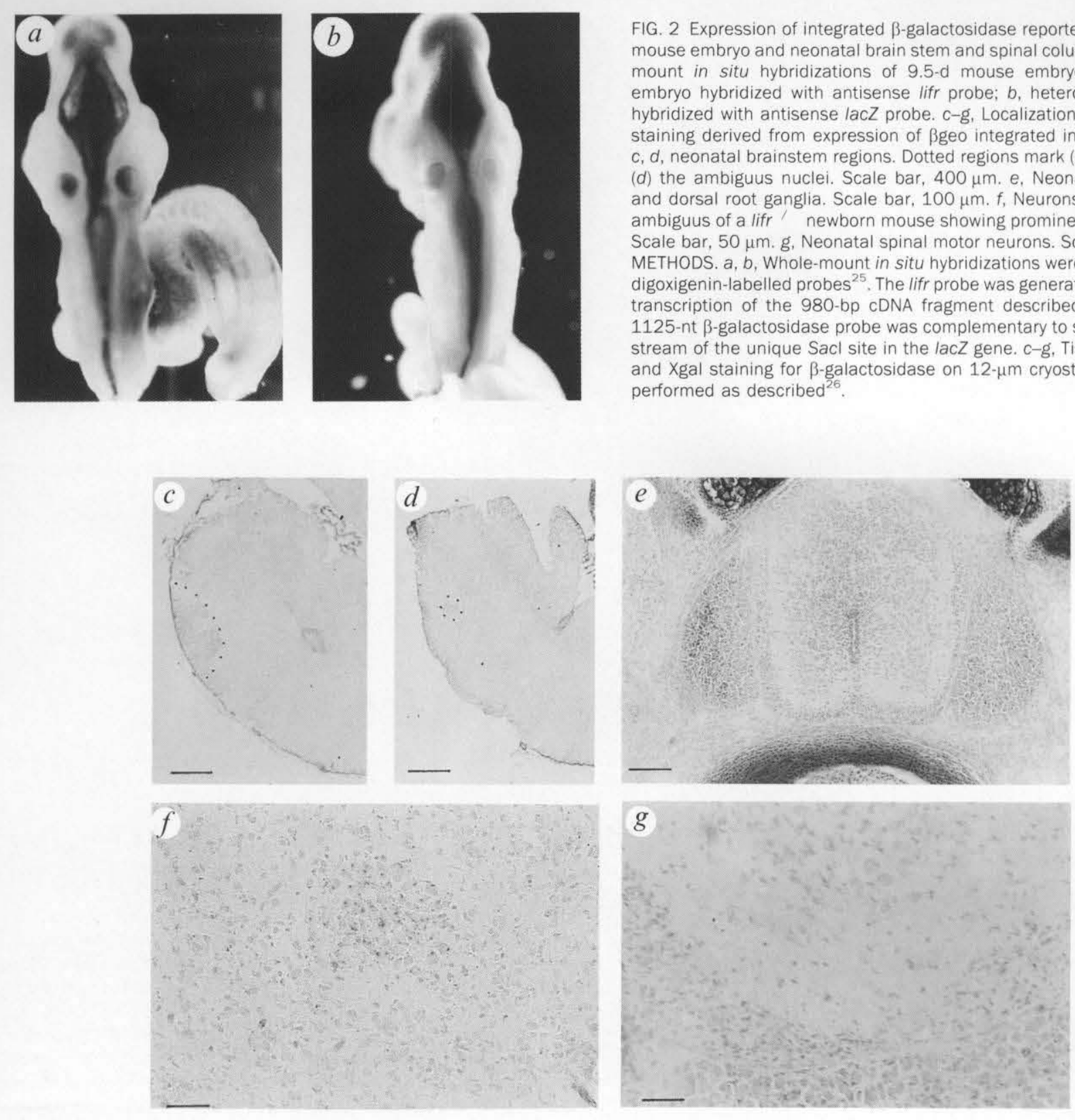

FIG. 2 Expression of integrated $\beta$-galactosidase reporter in the 9.5-day mouse embryo and neonatal brain stem and spinal column. $a, b$, Wholemount in situ hybridizations of 9.5-d mouse embryos: $a$, wild-type embryo hybridized with antisense lifr probe; $b$, heterozygous embryo hybridized with antisense lacZ probe. $c-g$, Localization of sites of Xgal staining derived from expression of Bgeo integrated into the lifr gene. $c, d$, neonatal brainstem regions. Dotted regions mark (c) the facial and (d) the ambiguus nuclei. Scale bar, $400 \mu \mathrm{m}$. e. Neonatal spinal cord and dorsal root ganglia. Scale bar, $100 \mu \mathrm{m}$. $f$, Neurons of the nucleus ambiguus of a lifr' newborn mouse showing prominent Xgal staining. Scale bar, $50 \mu \mathrm{m}$. g, Neonatal spinal motor neurons. Scale bar, $25 \mu \mathrm{m}$. METHODS. $a, b$, Whole-mount in situ hybridizations were made by using digoxigenin-labelled probes ${ }^{25}$. The lifr probe was generated by antisense transcription of the 980-bp cDNA fragment described in Fig. 1. The 1125 -nt $\beta$-galactosidase probe was complementary to sequence downstream of the unique $\mathrm{Sacl}$ site in the lacZ gene. $c-g$. Tissue processing and Xgal staining for $\beta$-galactosidase on $12-\mu \mathrm{m}$ cryostat sections was performed as described ${ }^{26}$.
FIG. 1 Targeted distribution of the lifr gene. a, Deletion strategy. Top, schematic representation of LIF-R protein showing the two cytokine receptor domains boxed. Vertical lines indicate the conserved cysteine residues, and solid bars represent the WSXWS motifs. Bottom, partial genomic structure of the lifr gene showing homology arms (thick lines) included in the targeting vector, positions of EcoRI restriction sites and sizes of diagnostic restriction fragments. Filled circles indicate the positions of flanking probes used to confirm replacement targeting events. The deletion region contains seven exons encoding amino acid residues 85-444 in the cytokine receptor domains of LIF-R. This region was replaced by an en-2 splice acceptor-IRES-ßgeopA cassette ${ }^{12}$, flanked by EcoRI restriction sites. $b$, Hybridization analysis of tail DNA from wildtype, heterozygous and homozygous lifr mutant mice. DNA samples were digested with EcoRI and sequentially hybridized with a $5^{\prime}$ genomic probe external to the targeting construct (left) and a cDNA fragment (right). The genomic probe was an 800-bp EcoRI/BamHI fragment shown in a. The cDNA probe was an internal 980-bp fragment that contains a sequence from the deleted exons 3-9 plus a sequence from exon 10. The 9.3-kb band present in heterozygotes and homozygotes corresponds to the recombinant junction fragment that includes exon 10. METHODS. a, lifr genomic clones were isolated from a 129Sv genomic library and the positions of exons determined (M.L., A.S., A. Cozens and I. Chambers, unpublished results). A promoterless targeting vector was constructed by subcloning the $5.5-\mathrm{kb}$ BamHI/Sall $5^{\prime}$ fragment and the 5.0-kb Smal/Sacl 3' fragment into pGEMEM (Promega) and inserting the GTIRES- $\beta$ geopA cassette. The construct was excised from the plasmid backbone by digestion with Notl and Sfil and electroporated into CGR8 ES cells maintained in the absence of feeders ${ }^{23}$. After selection in G418 the correct replacement event was identified in 19/58 colonies by filter hybridization with both 5 ' and 3 ' flanking probes. Single integration events were confirmed by hybridization with an en-2 probe. $b$, Two clones injected into $\mathrm{C} 57 \mathrm{BL} / 6$ blastocysts gave rise to germline transmission of the mutated allele. Animals were typed by hybridization analysis of tail DNA and/or by $\beta$-galactosidase-staining of ear biopsies ${ }^{24}$. Heterozygous offspring were backcrossed to the appropriate parental strain before intercrossing at the F2 and F3 generations. Homozygous offspring were identified and the deletion was confirmed by DNA filter hybridization. 
The differentiation and survival of motor neurons in vitro 4.79 and in vivo ${ }^{5.6 .10}$ have been shown to be strongly enhanced by CNTF and LIF, indicative of a requirement for LIF-R function. The inert condition of LIF-R-deficient neonates was suggestive of possible neuronal dysfunction. Therefore expression of LIF-R within the nervous system and the effects of gene ablation on motor neuron populations were investigated in more detail.

The fidelity of the reporter integrated into the lifi gene was established by the close similarity between hybridization patterns obtained in situ with antisense LIF-R and $\beta$-galactosidase probes and the correspondence observed between presence of IRES$\beta$ geo fusion transcripts and histochemically detectable $\beta$-galactosidase activity (Fig. 2, and results not shown). Expression in the developing nervous system was apparent in the hindbrain, cranial neural crest and neural tube of 9.5-day embryos (Fig. $2 a, b)$. The distribution of $\beta$-galactosidase activity in embryonic (14.4-day), neonatal and adult brain was then examined. Specific staining was evident in cells of the subependymal zone, ependyme and glia limitans. Expression was prominent in neurons in the brainstem motor nuclei, including the hypoglossal and facial nuclei (Fig. 2c, and results not shown), and also in the nucleus ambiguus (Fig. $2 d, f$ ). In sections through embryonic and neonatal spinal column, $\beta$-galactosidase staining was most pronounced in sensory neurons of the dorsal root ganglia but could also be detected in most of the spinal motor neurons (Fig. $2 e, g$, and results not shown). In addition some non-neuronal cells were stained, notably astrocytes of the glia limitans in the central nervous system and probably also Schwann cells in peripheral nerves.

The significance of the expression of LIF-R in spinal motor neurons and brainstem motor nuclei was investigated by counting motor neurons in the lumbar (L1-L6) spinal cord and facial nuclei of neonatal homozygous, heterozygous and wildtype littermates. The high expression of the $\beta$-galactosidase reporter found in the nucleus ambiguus also prompted an analysis of this structure. Motor neurons within the nucleus ambiguus innervate the oesophagus, pharynx and larynx, and coordinate swallowing. In addition there are neurons in the nucleus ambiguus of rats and mice that generate respiratory rhythm ${ }^{14-16}$. Data presented in Fig. 3 and Table 1 show that the numbers of motor neurons in LIF-R-deficient animals were reduced by $>35 \%$ in the facial nucleus and by $>40 \%$ in the lumbar spinal cord. The nucleus ambiguus neurons were reduced by over $50 \%$. Increased numbers of apparently dying cells with pyknotic nuclei were evident in both spinal-cord and brainstem
TABLE 1 Numbers of spinal motor neurons and neurons in facial and ambiguus nuclei of newborn wild-type, heterozygous and LIF-R-deficient mice

\begin{tabular}{lccc}
\hline & $+/+$ & +- & $-/-$ \\
Lumbar region (L1-L6) & $\begin{array}{c}3,213 \pm 230 \\
(n=3)\end{array}$ & N.D. & $\begin{array}{c}1,856 \pm 251 \\
(n=5)\end{array}$ \\
Facial nucleus & $3,108 \pm 310$ & $3,377 \pm 394$ & $1,951 \pm 135$ \\
& $(n=6)$ & $(n=5)$ & $(n=11)$ \\
Nucleus ambiguus & $883 \pm 82$ & $705 \pm 34$ & $406 \pm 32$ \\
& $(n=5)$ & $n=3)$ & $(n=5)$ \\
\hline
\end{tabular}

The lumbar spinal cord and brainstems of newborn mice or mice delivered by caesarian section at day 20 after conception were fixed by transcardial perfusion or immersion fixation with $4 \%$ paraformaldehyde. Paraffin serial sections $(7 \mu \mathrm{m})$ were prepared and processed as described $^{5,6}$. After Nissl staining, the number of neurons was counted in every tenth section of the lumbar (L1-L6) region of the spinal cord and every fifth section of the brainstem nuclei. Only neurons with distinguishable nucleus and nucleolus and with clearly identifiable Nissl structure were counted. Average diameters of the nucleoli were as follows. Facial motor neurons: $+/+$ mice, $2.64 \pm 0.43 \mu \mathrm{m} ;+/-$ mice, $2.32 \pm 0.22 \mu \mathrm{m} ;-/-$ mice, $2.14 \pm 0.16 \mu \mathrm{m}$; neurons of the nucleus ambiguus: $+/+$ mice, $2.36 \pm 0.36 \mu \mathrm{m} ;-/$ - mice, $2.34 \pm 0.41 \mu \mathrm{m}$ (means $\pm s . d$. for at least five determinations). The counts of facial and ambiguus neuronal nucleoli were corrected for double counting of split nucleoli. Spinal motor-neuron counts are uncorrected. N.D. not determined. Values shown are means \pm s.e.m. of each group. Numbers of neurons are different between $+/+$ and $-/-$ mice for each group $(P<0.005$, Student's t-test).

nuclei in paraffin sections from homozygotes (Fig. $3 d$, and results not shown). This suggests that elevated cell death of motor neurons is taking place around the time of birth.

Loss of facial motor neurons should not affect the viability of newborn mice. Impairment of the nucleus ambiguus is of greater significance. These neurons coordinate movements of the oesophagus and pharynx and are therefore required for suckling. Integrity of the nucleus ambiguus is also essential for generation of respiratory rhythm ${ }^{16}$. It is probable that loss of these neurons is a contributory factor to the early mortality observed in LIF$\mathrm{R}$-deficient mice. It would be of interest to know whether this structure is affected in other mouse mutants that die shortly after birth and exhibit reduced numbers of neurons in other brainstem nuclei.
FIG. 3 Neuronal morphology in the facial nucleus of newborn wild-type and lifr mutant mice. Facial nucleus of wildtype $(a, c)$ and homozygous lifr mutant $(b, d)$ newborn mice from the same litter. Scale bars: $a, b, 100 \mu \mathrm{m} ; c, d$, $25 \mu \mathrm{m}$.

METHODS. Paraffin sections $(7 \mu \mathrm{m})$ were prepared from brainstems of control and lifr $^{-1}$ mice and Nissl stained $^{5.6}$.
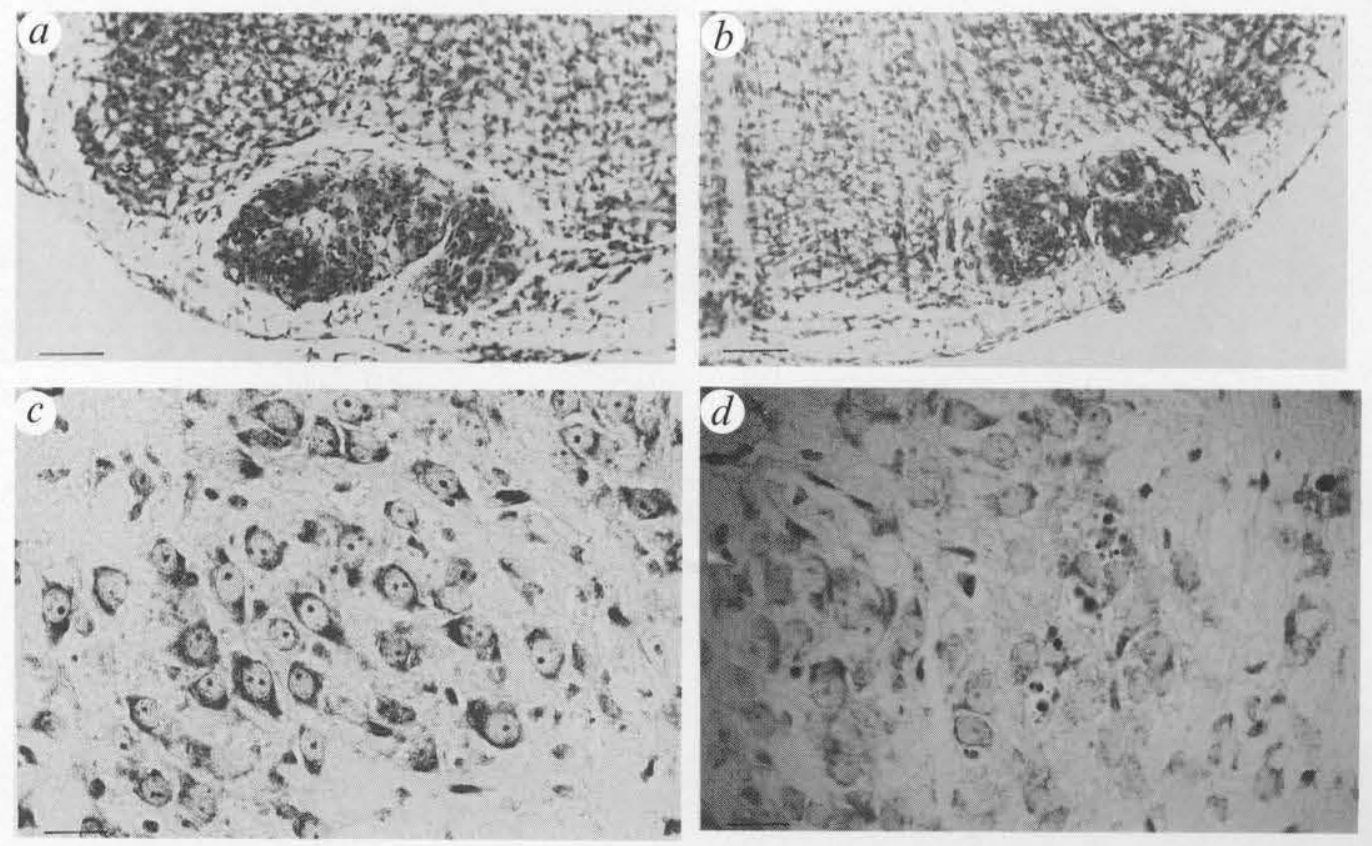
The motor-neuron losses and deficits in the nucleus ambiguus of LIF-R-deficient mice establish that these neurons are dependent on signalling through this receptor during embryonic development. Mice lacking CNTF do not show loss of motor neurons before adulthood ${ }^{6}$ and no severe neuronal defects have been observed in LIF-deficient mice (refs 17, 18 and M.S., unpublished observations). Mice deficient for both CNTF and LIF are viable and do not show functional motor defects at birth (M.S., unpublished observations). The lesions present in newborn LIF-R-deficient animals must therefore reflect the activity of one or more distinct cytokines

\section{Received 21 July; accepted 27 October 1995.}

1. Gearing, D. P. et al. EMBO J. 10, 2839-2848 (1991).

2. Gearing, D. P. et al. Science 255, 1434-1437 (1992)

3. Davis, S. et al. Science 260, 1805-1808 (1993).

4. Arakawa, Y. Sendtner, M. \& Thoenen, H. J. Neurosci. 10, 3507-3515 (1990).

5. Sendtner, M., Kreutzberg, G. W. \& Thoenen, H. Nature 345, 440-441 (1990).

6. Masu, Y. et ai. Nature 365, 27-32 (1993).

7. Richards, L. J., Kilpatrick, I. J., Bartlett, P. F. \& Murphy, M. J. Neurosci. Res. 33, 476-484 (1992).

8. Martinou, J.C., Martinou, I. \& Kato, A. C. Neuron 8, 737-744 (1992).

9. Zurn, A. D. \& Werren, F. Devi Biol. 163, 309-315 (1994).

10. Hughes, R. A., Sendtner, M. \& Thoenen, H. J. Neurosci. Res. 36, 663-671 (1993).

11. Ware, C. B. et al. Development 121, 1283-1299 (1995).

12. Mountford, P. et al. Proc. natn. Acad. Sci. U.S.A. 91, 4303-4307 (1994).

13. Mountford, P. \& Smith, A. G. Trends Genet. 11, 179-184 (1995)

14. Ellenberger, H. H. \& Feldman, J. L. J. comp. Neurol. 294, 202-211 (1990).

15. Smith, J. C., Greer, J. J., Liu, G. \& Feldman, J. L. J. Neurophysiol. 64, 1149-1169 (1990). active during embryogenesis. Candidates include the recently described cardiotrophin-1 (ref. 19) and a putative mouse orthologue of oncostatin $\mathrm{M}^{20}$. Interaction with the ligand may also involve the CNTF-receptor $\alpha$ (CNTF-R $\alpha$ ) subunit, which associates with LIF-R after CNTF binding ${ }^{3}$. CNTF-R $\alpha$ is expressed in the developing nervous system, in particular in motor neurons ${ }^{21}$, and a preliminary report indicates that its deletion results in developmental defects more severe than produced by deletion of $\mathrm{CNTF}^{22}$. The unidentified cytokine may therefore be a novel ligand for CNTF-R $\alpha$ in addition to LIF-R.
16. Paton, J. F. R., Ramirez, J.-M. \& Richter, D. W. Pflügers Arch. 428, 250-260 (1994). 17. Stewart, C. L. et al. Nature 359, 76-79 (1992)

18. Escary, J.-L., Perreau, J., Duménil, D., Ezine, S. \& Brûlet, P. Nature 363, 361-364 (1993) 19. Pennica, D. et al. J. biol. Chem. 270, 10915-10922 (1995).

20. Rose, T. M. \& Bruce, G. A. Proc. natn. Acad. Sci. U.S.A. 88, 8641-8645 (1991)

21. Ip, N. Y. et al. Neuron 10, 89-102 (1993)

22. Stahl, N. \& Yancopoulos, G. D. J. Neurobiol. 25, 1454-1466 (1994).

23. Smith, A. G. J. Tiss. Cult. Meth. 13, 89-94 (1991).

24. Beddington, R. S. P., Morgenstern, J., Land, H. \& Hogan, A. Development 106, 37-46 (1989).

25. Wilkinson, D. G. In Situ Hybridization: a Practical Approach (IRL, Oxford, 1992) 26. Skarnes, W. C., Auerbach, B. A. \& Joyner, A. L. Genes Dev. 6, 903-918 (1992).

ACKNOWLEDGEMENTS. M.L. and M.S. contributed equally to this work. We thank 1. Chambers for LIF-R cDNA, J. Nichols and J. Ure for chimaera production, A. Jeske for expert animal husbandry, and $H$. Döppler for excellent technical assistance; T. Taga for discussion, and I. husbandry, and $\mathrm{H}$. Doppler for excellent technical assistance; T. Taga for discussion, and $\mathrm{T}$. Chambers and $\mathrm{H}$. Niwa for comments on the manuscript. This research was supported by the
Biotechnology and Biological Sciences Research Council, UK, and the Deutsche Forschungsgemeinschaft, Germany. 